CERN/ECP 96-06

23 May 1996

\title{
RADIATION HARDNESS OF SILICON DETECTORS MANUFACTURED ON WAFERS FROM VARIOUS SOURCES
}

\author{
B. Dezillie, S. Bates, M. Glaser, F. Lemeilleur \\ CERN, Geneva, Switzerland \\ C. Leroy \\ University of Montreal, Montreal, Canada
}

\begin{abstract}
Impurity concentrations in the initial silicon material are expected to play an important role for the radiation hardness of silicon detectors, during their irradiation and for their evolution with time after irradiation. This work reports on the experimental results obtained with detectors manufactured using various float-zone (FZ) and epitaxially grown material. Preliminary results comparing the changes in leakage current and full depletion voltage of FZ and epitaxial detectors as a function of fluence and of time after $10^{14} \mathrm{~cm}^{-2}$ proton irradiation are given. The measurement of charge collection efficiency for epitaxial detectors is also presented.
\end{abstract}

Presented at the International Conference on Radiation Effects on Semiconductor Materials, Detectors and Devices, 6-8 March 1996, Florence, Italy. 
The study and the quest for new radiation-hard silicon detectors has become very active in the last few years. Because of their high efficiency, small thickness and fast readout, silicon detectors will be widely used in experiments at the future Large Hadron Collider (LHC). The LHC will bring proton beams into collision at centre-of-mass energies up to $14 \mathrm{TeV}$. The very high luminosities forseen $\left(\sim 10^{34} \mathrm{~cm}^{-2} \mathrm{~s}^{-1}\right)$ imply that silicon detectors have to resist hadron fluxes of the order of $10^{13} \mathrm{~cm}^{-2} \mathrm{yr}^{-1}$. Extensive studies have been performed to understand the characteristics of silicon detectors after irradiation [1,2]. This work presents preliminary results comparing float-zone (FZ) and epitaxial material which may open new perspectives.

The experimental conditions, the irradiation facilities and the measurement techniques are presented in Section 2. Results are given in Section 3.

\section{EXPERIMENTAL CONDITIONS}

\subsection{Test structures}

Standard diodes processed by Canberra, Intertechnique and Micron Semiconductor from n-type FZ materials typically have an area of $\sim 1 \mathrm{~cm}^{2}$ and a thickness of $\sim 300 \mu \mathrm{m}$.

The n-type epitaxial diodes were processed by Canberra. The epitaxial layer is grown on a n-type Czochralski base with a resistivity of $\sim 1 \Omega \mathrm{cm}$. The epitaxial detectors and their characteristics are listed in Table 1.

\section{Table 1}

Characteristics of epitaxial detectors used in this study. $\mathrm{V}_{\mathrm{fd}, 0}$ is the value of the full depletion voltage before irradiation.

\begin{tabular}{|c|c|c|c|c|c|}
\hline Detector name & $\begin{array}{c}\text { Thickness } \\
{[\mu \mathrm{m}]}\end{array}$ & $\begin{array}{c}\text { Area } \\
{\left[\mathrm{cm}^{2}\right]}\end{array}$ & $\begin{array}{c}V_{\text {fd.0 }} \\
{[\mathrm{V}]}\end{array}$ & $\begin{array}{c}\text { Resistivity } \\
{[\Omega \mathrm{cm}]}\end{array}$ & $\begin{array}{c}\Phi \\
\mathrm{cm}^{-2}\end{array}$ \\
\hline $\mathrm{C} 62$ & 110 & 0.82 & 43 & 860 & non irr \\
\hline $\mathrm{C} 63$ & 110 & 0.82 & 45 & 860 & $5.7 \times 10^{13} \mathrm{n}$ \\
\hline $\mathrm{C} 50$ & 120 & 0.82 & 48 & 960 & $1.3 \times 10^{14} \mathrm{p}$ \\
\hline
\end{tabular}

\subsection{Irradiation facilities}

Proton irradiations were performed at the CERN Proton Synchroton (PS) using the PST7 $24 \mathrm{GeV} / c$ primary beam which produces several bursts per minute providing an average flux of $\sim 3 \times 10^{9} \mathrm{p} \mathrm{cm}^{-2} \mathrm{~s}^{-1}$.

The neutron irradiations were performed at CERN using the Proton Synchroton Antiproton Irradiation Facility (PSAIF) which delivers bunches of $\sim 1 \mathrm{MeV}$ neutrons at a flux of $\sim 5 \times 10^{8} \mathrm{n} \mathrm{cm}^{-2} \mathrm{~s}^{-1}$.

\subsection{Measurement techniques}

Electrical characteristics were obtained from the current-voltage (IV) and capacitancevoltage (CV) measurements performed with a Keithley 237 high-voltage source measuring unit 
and a Hewlett-Packard 4194A impedance analyser operated at $10 \mathrm{kHz}$. The full depletion voltage is deduced from the measurement of the capacitance-versus-voltage (CV) curve and normalized to a $300 \mu \mathrm{m}$ diode thickness. The leakage current is obtained from the measurement of the current-versus-voltage (IV) curve at full depletion, normalized to $20{ }^{\circ} \mathrm{C}$ and corrected for self-annealing.

After irradiation, the diodes were stored at $20{ }^{\circ} \mathrm{C}$ and measured regularly during the first 100 days. After this time, they were heated for 1-3 hours at a maximum temperature of $90{ }^{\circ} \mathrm{C}$ in order to accelerate the annealing process using the model described in Ref. [2].

Electrons from a ${ }^{106} \mathrm{Ru}$ source with energies greater than $2 \mathrm{MeV}$ (m.i.p.), selected by an external trigger, were used for charge collection measurements. The pulse induced by an electron in a diode is detected by a Phillips Scientific model 6954 fast current amplifier and recorded on a digital oscilloscope operated in average mode.

\section{RESULTS}

\subsection{Leakage current}

\subsubsection{Leakage current versus fluence}

The change in volume leakage current with proton fluence is shown in Fig. 1. All data are normalized to $20^{\circ} \mathrm{C}$ and corrected for self-annealing in order to compare measurements taken at different times after irradiation.

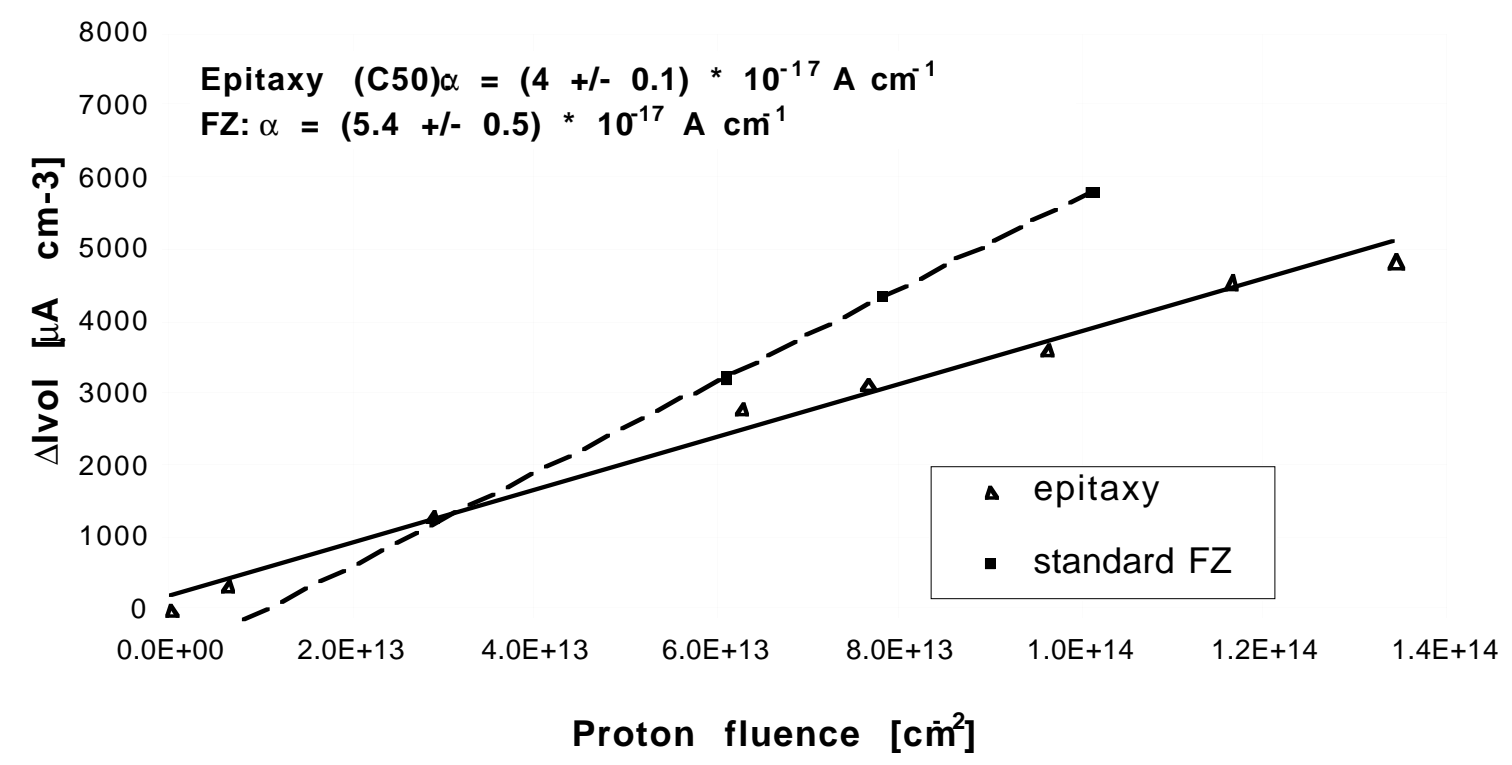

Fig. 1 The change in volume leakage current versus proton fluence. The leakage current has been normalized to $20^{\circ} \mathrm{C}$ and corrected for self-annealing. The solid line is a fit to the data obtained from an epitaxial detector. The dotted line is the average of separate fits to the different FZ detector data sets. 
The leakage current damage constant $\alpha$ is obtained from fitting to the form:

$$
\Delta I_{\mathrm{vol}}=\alpha \cdot \Phi
$$

where $\Delta I_{\mathrm{vol}}$ is the change in leakage current normalized to the depletion volume and $\Phi$ is the proton fluence. The solid line is the fit to the data of the epitaxial detector (C50), which gives an $\alpha$ value of:

$$
\alpha_{\text {epi }}=(4.0 \pm 0.1) \cdot 10^{-17} \mathrm{~A} \mathrm{~cm}^{-1} \text {. }
$$

On the same figure is shown, for comparison, the data obtained for a standard FZ detector. The dotted line is the average of fits to different detector data, separately [3]. The $\alpha$ value is:

$$
\alpha_{\mathrm{FZ}}=(5.4 \pm 0.5) \cdot 10^{-17} \mathrm{~A} \mathrm{~cm}^{-1} \text {. }
$$

\subsubsection{Leakage current versus time after irradiation}

The behaviour of the leakage current with time after irradiation is shown in Fig. 2 for two epitaxial detectors (C50 and C63) and for a standard FZ detector. The value of $\alpha$ is shown, rather than the leakage current, to normalize for differences in proton and neutron fluences between the different detectors. Like standard FZ diodes, the currents of the epitaxial detectors decrease regularly and stabilize at a value of about $1 \times 10^{-17} \mathrm{~A} \mathrm{~cm}^{-1}$ for $\alpha$, which is slightly lower than the $\alpha$ value for the FZ detectors.

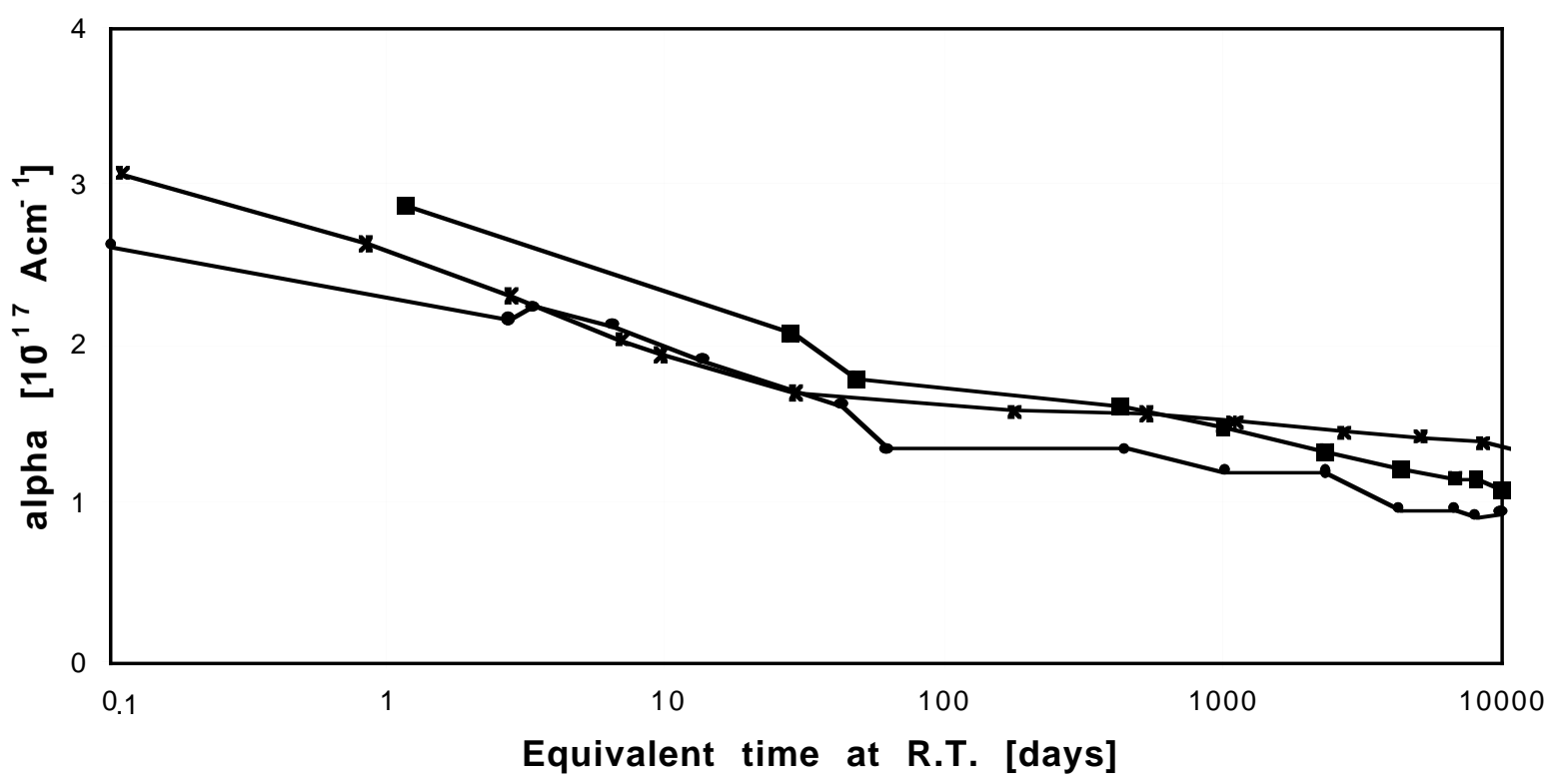

Fig. 2 Leakage current damage constant as a function of equivalent time at R.T. for a $5.7 \times 10^{13} \mathrm{~cm}^{2}$ neutron irradiated epitaxial $(\mathrm{n})$, a $1.3 \times 10^{14} \mathrm{~cm}^{2}$ proton irradiated epitaxial detector (1), and a $1 \times 10^{14} \mathrm{~cm}^{2}$ proton irradiated FZ detector (*). 


\subsection{Depletion voltage}

\subsubsection{Depletion voltage versus fluence}

Figure 3 shows the full depletion voltage normalized to a thickness of $300 \mu \mathrm{m}$ as a function of proton fluence for an epitaxial diode and for FZ detectors with different initial resistivities and from different manufacturers. Measurements are taken within one hour after each irradiation step so that long-term annealing can be neglected and all data are normalized to a thickness of $300 \mu \mathrm{m}$.

At $1.6 \times 10^{13}$ protons $\mathrm{cm}^{-2}$, one high-resistivity $\mathrm{FZ}$ detector is slightly inverted, while all the others are not yet inverted. At $5.1 \times 10^{13}$ protons $\mathrm{cm}^{-2}$, detectors marked with dashed lines are slightly inverted. Detectors marked with dotted lines are strongly inverted when they reach the fluence value of $10^{14}$ protons $\mathrm{cm}^{-2}$.

While inversion from n- to p-type is observed for FZ diodes, no inversion is seen for the epitaxial detector irradiated up to the level of $1.3 \times 10^{14} \mathrm{~cm}^{-2}$. Using $\Phi_{\text {inv }}=(18 \pm 0.6) N_{\text {eff, } 0}[3]$ for FZ detectors, the inversion should happen at $\sim 8 \times 10^{13} \mathrm{~cm}^{-2}$. This preliminary result of noninversion of an epitaxial detector after a fluence of $1.3 \times 10^{14} \mathrm{~cm}^{-2}$ is an important result for the future LHC experiments.

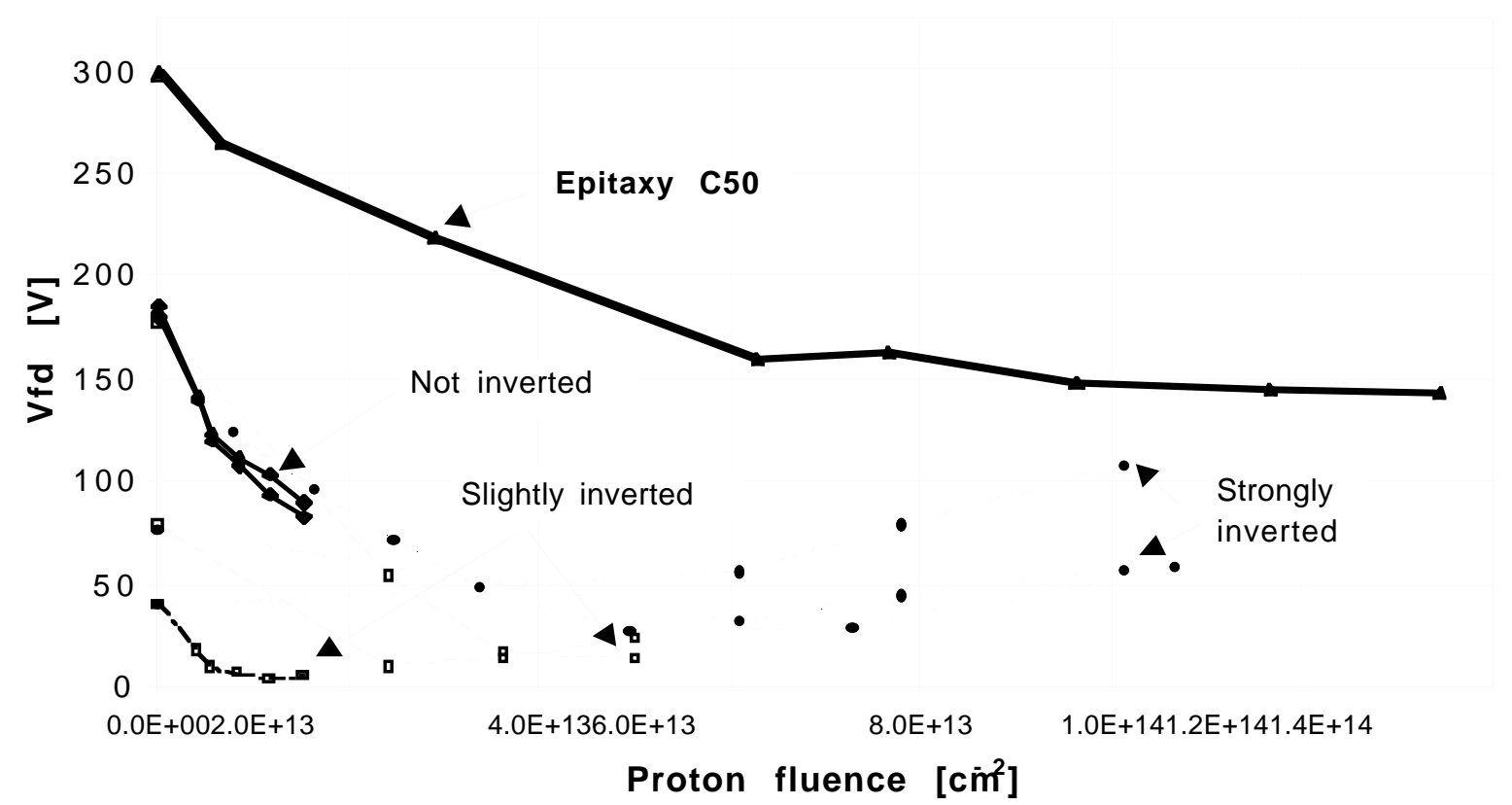

Fig. 3 Depletion voltage (normalized to a thickness of $300 \mu \mathrm{m}$ ) as a function of fluence. No inversion is observed for epitaxial detectors. 


\subsubsection{Depletion voltage versus time after irradiation}

Figure 4 shows the full depletion voltage (normalized to a thickness of $300 \mu \mathrm{m}$ ) as a function of equivalent time at room temperature for three sets of FZ detectors irradiated at $1.6 \times 10^{13}, 5.1 \times 10^{13}$ and $1.2 \times 10^{14}$ protons $\mathrm{cm}^{-2}$ and for the epitaxial detector irradiated at $1.3 \times 10^{14}$ protons $\mathrm{cm}^{-2}$. The two FZ detectors and the epitaxial detector which were not inverted (solid lines) show no reverse annealing. A strong reverse annealing is observed for strongly inverted detectors (dotted lines), while slightly inverted detectors (dashed lines) have an intermediate behaviour at room temperature.

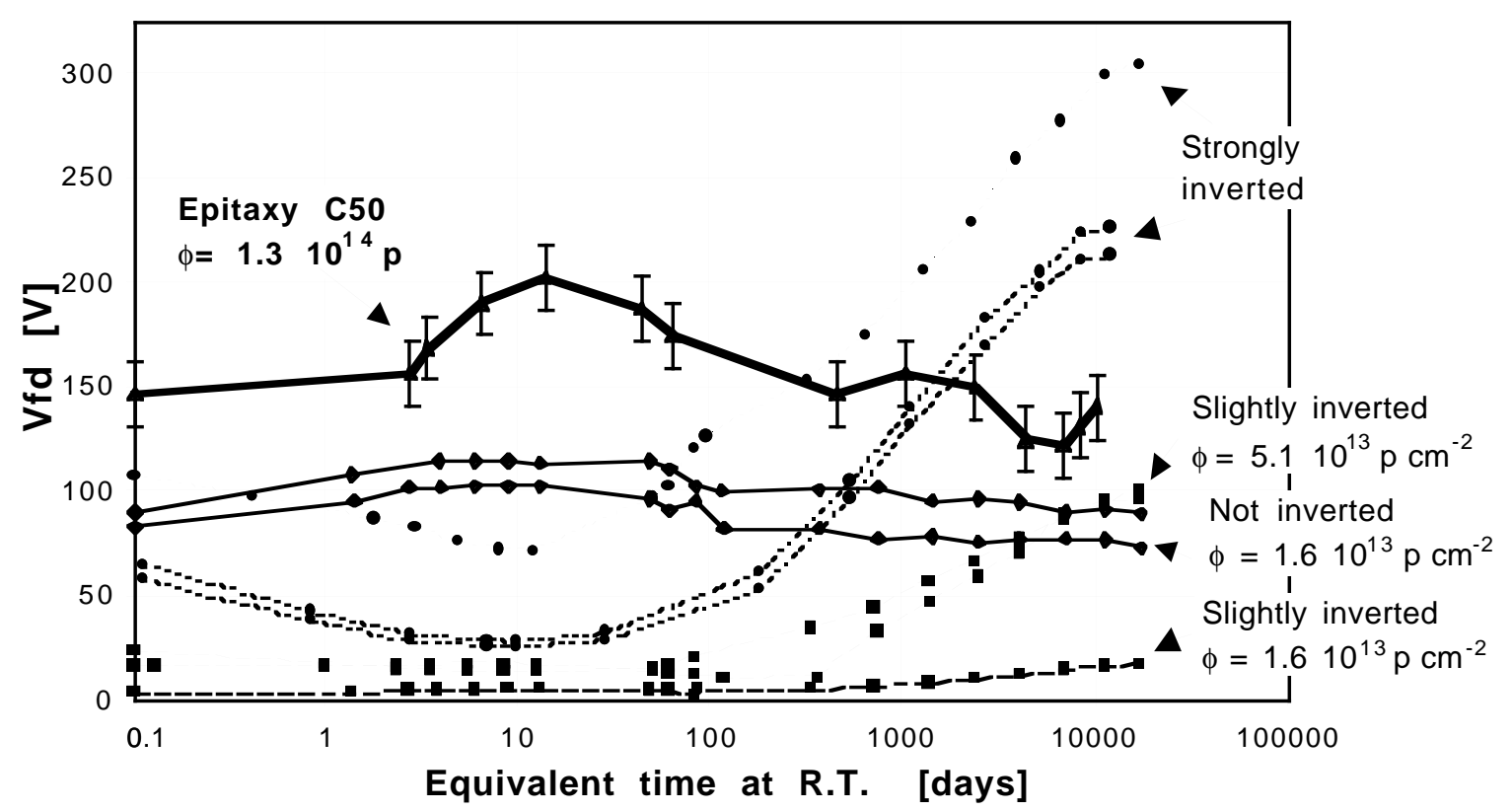

Fig. 4 Depletion voltage (normalized to a thickness of $300 \mu \mathrm{m}$ ) as a function of equivalent time at room temperature after irradiation. Non-inverted detectors show no reverse annealing.

\subsection{Charge collection}

Figure 5 shows the average charge collection of epitaxial detectors as a function of $V^{1 / 2}$ for a non-irradiated detector C62 $\left(Q_{0}\right)$, and for an irradiated detector C63 $\left(Q_{\text {irr }}\right)$ with neutrons at a fluence of $5.7 \times 10^{13} \mathrm{~cm}^{-2}$. The charge collection efficiency $Q_{\text {irr }} / Q_{0}$ is $(92.7 \pm 3.2) \%$. This corresponds to an efficiency of $87.2 \%$ for a fluence of $10^{14} \mathrm{n} / \mathrm{cm}^{2}$, which is in good agreement with the efficiency of $(88 \pm 4) \%$ measured for standard FZ detectors [4].

On the same figure is also plotted the average charge collection for a $306 \mu \mathrm{m}$ thick $\mathrm{FZ}$ detector M165, non-irradiated. It can be observed that for non-irradiated detectors the ratio of the average charge collection for FZ to epitaxial material is equal to the ratio of their relative thicknesses. 


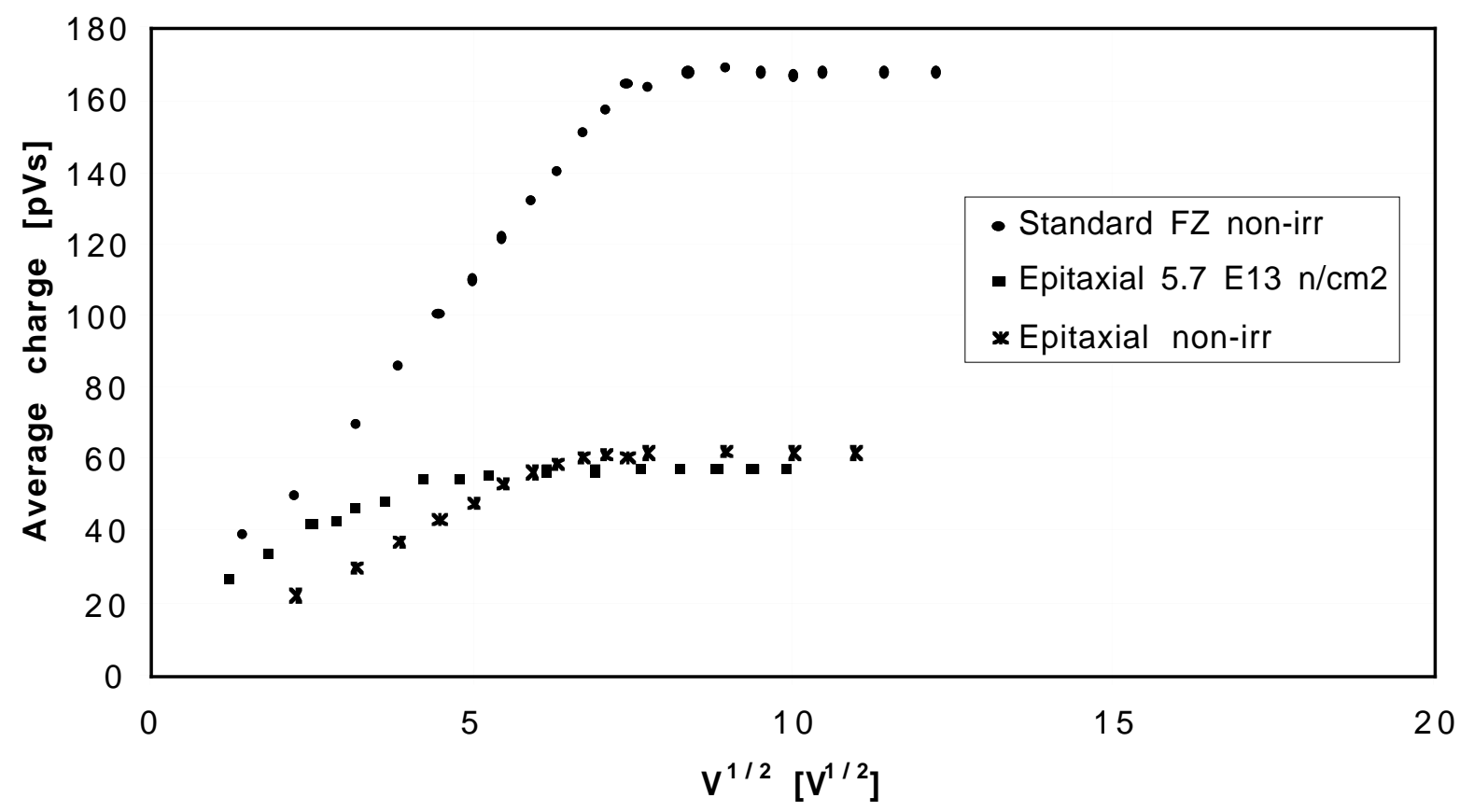

Fig. 5 The average charge collection as a function of $V^{1 / 2}$ for a non-irradiated epitaxial (C62), a $5.7 \times 10^{13} \mathrm{n} / \mathrm{cm}^{2}$ irradiated epitaxial (C63), and a non-irradiated standard $306 \mu \mathrm{m}$ thick FZ (M165) detector.

\section{CONCLUSION}

Preliminary results on epitaxial detectors have been presented. Epitaxial detectors (ntype) with resistivity of 860-960 $\Omega \mathrm{cm}$ and thickness of 110-120 $\mu \mathrm{m}$ have been studied and compared with standard (n-type) FZ detectors. They have been irradiated at room temperature with $1 \mathrm{MeV}$ neutrons and $24 \mathrm{GeV} / c$ protons. The value of the damage constant corrected for self-annealing during the proton irradiation for epitaxial detectors was found to be $\alpha=(4.0 \pm 0.1) 10^{-17} \mathrm{~A} \mathrm{~cm}^{-1}$, which is slightly lower than the value obtained for standard FZ detectors.

While the annealing at room temperature of the reverse current tends to saturate after several weeks for FZ detectors $\left(\alpha \sim 1.5 \times 10^{-17} \mathrm{~A} \mathrm{~cm}^{-1}\right)$, it has been found that the reverse current for the epitaxial detectors continues to decrease after a period of more than 10 years $\left(\alpha \sim 1 \times 10^{-17} \mathrm{~A} \mathrm{~cm}^{-1}\right)$.

Non-inverted FZ detectors show no voltage reverse annealing and the higher the fluence above the type inversion point, the greater the reverse annealing of depletion voltage.

No type inversion and no reverse annealing of depletion voltage are observed for the epitaxial detector studied up to $1.3 \times 10^{14}$ proton $\mathrm{cm}^{-2}$ irradiation. This study proves that there exists detector material such as the epitaxial type from which detectors can be manufactured. Such detectors can be operated fully depleted at room temperature at voltages $\leq 50 \mathrm{~V}$ for a thickness of $120 \mu \mathrm{m}$ up to a proton fluence of $10^{14} \mathrm{~cm}^{-2}$ during 10 years of operation at the LHC.

The charge collection efficiency for epitaxial detectors with a deficit of $\sim 8 \%$ after a neutron fluence of $5.3 \times 10^{13} \mathrm{~cm}^{-2}$ is similar to the deficit obtained for $\mathrm{FZ}$ detectors. 
This surprising behaviour during and after irradiation of diodes processed from epitaxially grown crystals is a confirmation that the radiation hardness of silicon depends on the impurity concentrations of the initial material [3]. Measurements of the impurity concentrations of the FZ and epitaxial material used for this study are under way. New measurements are forseen with epitaxial detectors with a higher resistivity and a larger thickness. A production of FZ silicon crystals with different impurity concentrations is also under way.

\section{REFERENCES}

[1] F. Lemeilleur et al., "Electrical properties and charge collection efficiency for neutronirradiated p-type and n-type silicon detectors", Nucl. Phys. B (Proc. Suppl.) 32 (1993) 415.

[2] E. Fretwurst et al., "Radiation hardness of silicon detectors for future colliders", Nucl. Intrum. Methods Phys. Res. A326 (1993) 357.

[3] S.J. Bates et al., "Proton irradiation of silicon detectors with different resistivities", CERN/ECP 95-18, to appear in the Proceedings of the IIIrd European Symposium Radiations and their Effects on Components and Systems, Arcachon, France, Sept. 1995.

[4] C. Leroy et al., "Study of electrical properties and charge collection of silicon detectors under neutron, proton and gamma irradiations", in Proc. IVth Int. Conf. on Calorimetry in High Energy Physics, La Biodola, Isola d'Elba, Italy, Eds. A. Menzione and Scribano (World Scientific, Singapore, 1994), p. 627. 